\section{D) Check for updates}

Cite this: Analyst, 2019, 144, 4559

\title{
Comparison between conventional and frit-inlet channels in separation of biopolymers by asymmetric flow field-flow fractionation
}

\author{
Catalina Fuentes, (D) *a,b Jaeyeong Choi, (D) a,c Claudia Zielke, (D) ${ }^{a}$ \\ J. Mauricio Peñarrieta, (D) ${ }^{b}$ Seungho Lee (D) ${ }^{c}$ and Lars Nilsson (D) ${ }^{a}$
}

\begin{abstract}
Asymmetric flow field-flow fractionation (AF4) is a separation technique in which a focusing/relaxation step is used after the sample is injected onto the separation channel. During the focusing/relaxation step, the sample is focused by two counter-directed flows. This allows sample components to establish a diffusion-dependent equilibrium concentration profile. The focusing step may, in some cases, cause a loss of sample due to adsorption into the accumulation wall (i.e. the membrane) or due to aggregation of the sample. In addition, the increase in sample concentration during the focusing step may prevent complete relaxation and cause overloading effects. In this study, a modified AF4 channel equipped with a frit inlet (FI-AF4) is utilized, where the sample is relaxed hydrodynamically as it enters to the channel through the frit. The main advantage of the FI-AF4 channel is to omit the focusing step. The FI-AF4 channel could also allow higher injection mass than in a conventional channel while still avoiding overloading. The purpose of the present study is to compare two channels (conventional and Fl-AF4 channels) in terms of the plate height $(H)$, resolution $\left(R_{\mathrm{s}}\right)$ and the mass recovery for analysis of a mixture of glycogen and pullulan. In addition, waxy maize (WM) starch was used to compare the mass overloading of the two channels. The results show that the type of relaxation method (i.e. focusing or hydrodynamic relaxation) had no significant effect on mass recovery. The resolution $\left(R_{\mathrm{s}}\right)$, was higher in the conventional AF4 channel than in the FI-AF4 channel for the separation of glycogen and pullulan. The results also show that it was possible to inject a higher mass of WM starch (i.e. twice the mass) onto the FI-AF4 channel, compared to a conventional AF4 channel, without observing an overloading effect.
\end{abstract}

Received 13th March 2019, Accepted 14th June 2019

DOI: 10.1039/c9an00466a

rsc.li/analyst channel. Therefore, different components emerge at different times and they can be detected or collected individually. ${ }^{1}$

Among the FFF sub-techniques, the most universal is the asymmetric flow field-flow fractionation (AF4). In this technique, a solid wall on the top and a permeable wall on the bottom form the separation channel. After the sample is injected onto the channel, an important step is the focusing/ relaxation of sample components. In this step, the injected sample is focused into a thin band by two counter-directed flows (one from the channel inlet and the other from the channel outlet), and is relaxed (reaches an equilibrium concentration profile) below the injection point of the sample. The relaxation is achieved through the applied perpendicular crossflow, which transports sample components towards the accumulation wall i.e. the bottom of the separation channel consisting of an ultrafiltration membrane. The transport is counteracted by the diffusion of the sample components and, at steady state, a crossflow and diffusion dependent concentration profile is established. At the accumulation wall, the components are confined to a thin relatively concentrated layer. The sample components with a higher diffusion coeffi- 
cient $(D)$ are, on average, distributed farther away from the accumulation wall than components with a lower $D$. After the focusing/relaxation step, the separation process begins by applying a longitudinal laminar flow of a carrier liquid through the separation channel in combination with the crossflow in a perpendicular direction over the channel. ${ }^{2}$ As the flow profile along the separation channel is parabolic, the components distributed farther away from the accumulation wall travel faster downstream, and size separation is achieved. ${ }^{3}$

The focusing/relaxation step might, in some cases, cause a loss of sample due to adsorption at the accumulation wall (i.e. the membrane) or aggregation of sample components, resulting in low sample recoveries. ${ }^{4}$ One way to avoid the focusing step is to use a modified channel, based on a hydrodynamic relaxation, using a frit inlet (FI). ${ }^{5,6}$ Another advantage of the FI-AF4 channel is that the injection mass of the sample could probably be higher than in the conventional channel since high concentrations during focusing/relaxation are, to some extent, avoided. This would be beneficial in the analysis of ultra-high molar mass $\left(>10^{7} \mathrm{~g} \mathrm{~mol}^{-1}\right)$ branched polymers like amylopectin, where overloading occurs already at very low injected mass in conventional AF4 channels. ${ }^{7,8}$ To avoid overloading and compromised separation, it can, thus, be necessary to inject very low sample mass. In turn, the low injected mass can complicate detection. This can especially be the case for large biopolymers, such as amylopectin, where concentration detection and molar mass determination relies on differential refractive index (dRI) detection.

After sample injection and relaxation (either focusing or hydrodynamic), the separation mechanism in the FI-AF4 channel is the same as that in a conventional AF4 channel, as described above. The difference is that, instead of having a dedicated injection port for the sample, the FI-AF4 channel has a frit through which a relatively higher carrier flow can pass. The hydrodynamic relaxation is achieved when faster frit flow meets slower sample flow, pushing the sample toward the accumulation wall. ${ }^{6}$ It has been shown that FI-AF4 can give higher separation channel mass recoveries in protein analyses. ${ }^{9,10}$ However, resolution was shown to be lower than for AF4 and analysis time was considerably longer. To date, FI-AF4 and AF4 have not been thoroughly compared for separation of hyper-branched ultra-high molar mass biopolymers such as amylopectin nor for mixtures of branched and linear biopolymers such as pullulan and glycogen.

In this study, the performance of a conventional AF4 and a FI-AF4 channel are compared in terms of the mass recovery, plate height $(H)$ and resolution $\left(R_{\mathrm{s}}\right)$. For this purpose, we use a mixture of biopolymers, i.e. glycogen and pullulan. The two polymers have overlapping molar mass $(M)$ range but different hydrodynamic size and structure, i.e. branched and linear, respectively. Pullulan was chosen as it is commonly used as a standard biopolymer and glycogen as it is hyper-branched, and relatively well defined. In addition, both biopolymers display high aqueous solubility. Furthermore, the overloading effect as a result of injected mass is investigated in both conventional AF4 and FI-AF4 channels using waxy maize (WM) starch which is, due to its ultra-high molar mass and hyperbranched structure, well-known for being sensitive to overloading effects in $\mathrm{AF} 4 .^{7,8}$

\section{Materials and methods}

\section{Materials}

Glycogen from bovine liver type IX with molar mass distribution from $3 \times 10^{5}$ to $10^{7} \mathrm{~g} \mathrm{~mol}^{-1}\left(M_{\mathrm{w}}=2.9 \times 10^{5} \mathrm{~g} \mathrm{~mol}^{-1}\right)$ and $z$-average root-mean-square radius $\left(r_{\mathrm{rms}}\right)$ of approx. $10 \mathrm{~nm}$ (ref. 11) was purchased from Sigma Aldrich (Darmstadt, Germany); pullulan with $M$ from $10^{5}$ to $10^{7} \mathrm{~g} \mathrm{~mol}^{-1}\left(M_{\mathrm{w}}=2.1 \times\right.$ $10^{5} \mathrm{~g} \mathrm{~mol}^{-1}$ ) and $z$-average $r_{\text {rms }}$ approx. $30 \mathrm{~nm}$ (ref. 11) was obtained from Guangzhou Medcan Pharmatech Ltd (Guangdong, China); bovine serum albumin (BSA) was purchased from Sigma Aldrich; $\mathrm{NaNO}_{3}$ (A3125) was from AppliChem (Darmstadt, Germany) and $\mathrm{NaN}_{3}$ (10369) was supplied by BDH (Poole, UK). Waxy maize (WM) (virtually 100\% amylopectin) was provided by Cerestar- (Vodsko, Denmark).

\section{Sample preparation}

Glycogen and pullulan solutions were prepared with AF4 carrier liquid at a concentration of 1 and $4 \mathrm{mg} \mathrm{mL}^{-1}$, respectively. The carrier liquid was $10 \mathrm{mM}$ of $\mathrm{NaNO}_{3}$ and $0.02 \%(\mathrm{w} / \mathrm{v})$ $\mathrm{NaN}_{3}$, dissolved in pure water from a Milli-Q system (Millipore Corp., Billerica, MA, USA) under stirring $(\mathrm{pH}=7)$. All samples were filtered through a $0.45 \mu \mathrm{m}$ cellulose acetate filter (PALL Life Sciences syringe filter, $25 \mathrm{~mm}$ diameter, Pall Corporation, Port Washington, NY, USA) prior to injection.

The effect of sample concentration in the mixtures of glycogen and pullulan was studied by mixing the solutions at different volume ratios, which correspond to their respective mass/mass ratios that are shown in Table 1 . The injection volume of the mixtures, in all cases, was $50 \mu \mathrm{L}$, which corresponds to sample mass shown in Table 1 . For the rest of the analysis, the samples were mixed at the ratio of $1: 4(\mathrm{w} / \mathrm{w})$.

The WM sample was prepared as described elsewhere, ${ }^{12}$ with a minor difference in the dilution step: the sample solution of $1 \mathrm{mg} \mathrm{mL}{ }^{-1}$ was diluted using the carrier liquid at $100{ }^{\circ} \mathrm{C}$ to various concentrations of $0.125,0.25,0.33,0.50$ and 0.75 $\mathrm{mg} \mathrm{mL}^{-1}$. The sample with $1 \mathrm{mg} \mathrm{mL}^{-1}$ was injected without dilution. The injection volume of the sample in all cases was $10 \mu \mathrm{L}$, corresponding to sample mass from 1.25 to $10 \mu \mathrm{g}$.

Table 1 Ratios of glycogen/pullulan (w/w) in the mixtures prepared to study the effect of sample concentration

\begin{tabular}{lll}
\hline Ratio glycogen/pullulan $(\mathrm{w} / \mathrm{w})$ & Glycogen $(\mu \mathrm{g})$ & Pullulan $(\mu \mathrm{g})$ \\
\hline $1.00: 6.00$ & 25 & 150 \\
$1.00: 5.00$ & 25 & 125 \\
$1.00: 4.00$ & 25 & 100 \\
$1.00: 2.00$ & 25 & 50 \\
$1.00: 1.00$ & 25 & 25 \\
$1.00: 0.50$ & 25 & 12.5 \\
$1.00: 0.25$ & 25 & 6.25
\end{tabular}




\section{Asymmetric flow field-flow fractionation (AF4)}

Samples were analyzed by AF4-MALS-dRI using an Eclipse 3+ System (Wyatt Technology, Dernbach, Germany) connected to a Dawn Heleos II multiangle light scattering (MALS) detector (Wyatt Technology) and an Optilab T-rEX differential refractive index (dRI) detector (Wyatt Technology). Both detectors operated at the wavelength of $658 \mathrm{~nm}$. An Agilent 1100 series isocratic pump (Agilent Technologies, Waldbronn, Germany) with an in-line vacuum degasser and an Agilent 1100 series autosampler delivered the carrier flow and handled sample injection onto the AF4 separation channel. A filter holder with a polyvinylidene fluoride membrane of pore size $100 \mathrm{~nm}$ (Millipore Corp.), was placed between the pump and the channel to ensure that particle-free carrier liquid entered to the system.

\section{Separation channels}

The conventional AF4 channel was a "long" channel (Wyatt Technology) with a trapezoidal shape (tip-to-tip length of $26.5 \mathrm{~cm}$ and inlet and outlet widths of 2.6 and $0.6 \mathrm{~cm}$, respectively), equipped with a spacer with a nominal thickness of $350 \mu \mathrm{m}$.

The frit inlet (FI) AF4 channel (Wyatt Technology) was also trapezoidal with the same shape and dimensions as the conventional AF4 channel. The FI of the channel was $3.2 \mathrm{~mm}$ at the inlet end of the polycarbonate top of the channel. The spacer had a nominal thickness of $350 \mu \mathrm{m}$ with a sample inlet canal of $4.0 \mathrm{~mm} \times 1 \mathrm{~mm}$ between the frit and sample flow.

In both channels, the ultra-filtration membrane forming the accumulation wall was a regenerated cellulose membrane with a nominal cut-off of $10 \mathrm{kDa}$ (Merck Millipore, Bedford, MA, USA). The performance of the AF4 system, regarding size separation and molar mass determination was checked with bovine serum albumin (BSA) solution $\left(1 \mathrm{mg} \mathrm{mL} \mathrm{m}^{-1}, \mathrm{w} / \mathrm{v}\right)$ according to the procedure described elsewhere. ${ }^{13}$

\section{The effect of sample concentration}

For the analysis of a mixture of glycogen and pullulan, a constant channel flow $\left(Q_{\text {out }}\right)$ of $0.2 \mathrm{~mL} \mathrm{~min}^{-1}$ was used for both conventional AF4 and FI-AF4 channels. The sample injection into the channel was performed at the flow rate of $0.2 \mathrm{~mL} \mathrm{~min}^{-1}$ for $4 \mathrm{~min}$. In the case of FI-AF4, this time represents the initial relaxation time (i.e. hydrodynamic relaxation), where the initial crossflow rate $\left(Q_{\mathrm{c} 0}\right)$ is kept constant. For the conventional $\mathrm{AF} 4$ channel, after injection, a 4 min step of relaxation time (i.e. focus time) was applied, where two counter-directed flows focused the sample. This step was performed using a focusing flow rate equal to the $Q_{\mathrm{c} 0}$. The crossflow rate was programmed to decay linearly according to the following equation:

$$
Q_{\mathrm{c}}(t)=Q_{\mathrm{c}}(0)-\Delta Q_{\mathrm{c}}\left(\frac{t-t_{1}}{t_{\mathrm{p}}}\right)
$$

where $Q_{\mathrm{c}}(t)$ is the crossflow rate as a function of time $t$ after the elution starts, $Q_{\mathrm{c}}(0)$ is the initial crossflow rate, $t_{\mathrm{p}}$ is the transient time for linear decay and $t_{1}$ is the initial time delay, which is the period in which a constant flow rate is maintained before decay. The elution started at $Q_{\mathrm{c} 0}=1.5 \mathrm{~mL} \mathrm{~min}^{-1}$, which

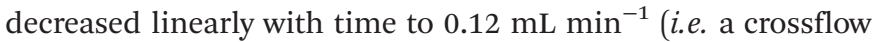
half-life of $6 \mathrm{~min}$ ), and then kept constant for $10 \mathrm{~min}$. Finally, the channel was flushed without any crossflow for $15 \mathrm{~min}$ before the next injection.

For the analysis of waxy maize starch (WM), $Q_{\text {out }}$ was kept constant at $1.5 \mathrm{~mL} \mathrm{~min}{ }^{-1}$, while the sample injection into the channel was performed at a flow rate of $0.2 \mathrm{~mL} \mathrm{~min}^{-1}$ for 4 min. Again, in the case of FI-AF4, this time represents the initial relaxation time with the initial crossflow rate $\left(Q_{\mathrm{co}}\right)$ kept constant.

For the conventional AF4 channel, after injection, a $4 \mathrm{~min}$ focusing/relaxation was performed at the focusing flow rate of $Q_{\mathrm{c} 0}$. The crossflow rate was programmed to decay exponentially according to the following equation:

$$
Q_{\mathrm{c}}(t)=Q_{\mathrm{c}}(0) e^{\left(-\frac{\ln 2}{t_{1 / 2}} t\right)}
$$

The elution started at $Q_{\mathrm{c} 0}$ of $2.5 \mathrm{~mL} \mathrm{~min}^{-1}$, which was decreased exponentially over time to $0.13 \mathrm{~mL} \mathrm{~min}^{-1}\left(t_{1 / 2}=\right.$ $4 \mathrm{~min}$ ), and then was maintained constant for $23 \mathrm{~min}$. The channel was flushed without any crossflow for $10 \mathrm{~min}$ before the next injection. It has been shown in previous studies that, for a successful separation in FI-AF4, the ratio of the sample inlet flow rate $\left(Q_{\mathrm{s}}\right)$ to the frit flow rate $\left(Q_{\mathrm{f}}\right)$, i.e. $Q_{\mathrm{s}} / Q_{\mathrm{f}}$, must be either less or equal to 0.05 to minimize band broadening during hydrodynamic relaxation. ${ }^{4,6,10}$ For this reason, the $Q_{\mathrm{s}} / Q_{\mathrm{f}}$ used for the analysis of WM was close to 0.05 , which means that it should be near to complete hydrodynamic relaxation.

\section{The effect of initial crossflow rate $\left(Q_{\mathrm{c} 0}\right)$ and focus/relaxation time}

The effect of $Q_{\mathrm{c} 0}$ on the mass recovery, plate height $(H)$ and resolution $\left(R_{\mathrm{S}}\right)$ was studied by comparing four different crossflow rate conditions as shown in Table 2 and Fig. 1. In all cases, the crossflow rate was programmed to decay linearly with the slope kept constant.

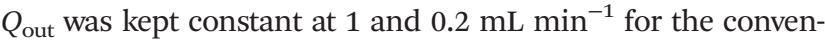
tional AF4 channel and the FI-AF4 channel, respectively. In the conventional AF4 channel, the sample was injected at a flow rate

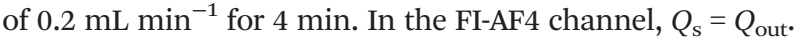

To examine the effect of the relaxation time on the mass recovery, plate height $(H)$ and resolution $\left(R_{\mathrm{s}}\right), Q_{\mathrm{co}}$ was kept constant for 2, 4 and $6 \mathrm{~min}$, respectively, before $Q_{\mathrm{c}}$ started decaying. For the conventional AF4 channel, this time corresponds

Table 2 Crossflow programing conditions used to study the effect of initial crossflow rate $\left(Q_{c 0}\right)$

\begin{tabular}{lllll}
\hline $\begin{array}{l}\text { No. } \\
\text { program }\end{array}$ & $\begin{array}{l}\text { Initial crossflow } \\
Q_{\mathrm{co}}\left(\mathrm{mL} \mathrm{min}{ }^{-1}\right) \\
\text { at } t=0\end{array}$ & $\begin{array}{l}\text { Final } \\
\text { crossflow } Q_{\mathrm{cf}} \\
\left.(\mathrm{mL} \mathrm{min})^{-1}\right)\end{array}$ & $\begin{array}{l}t_{1}+t_{\mathrm{p}}{ }^{a} \\
(\mathrm{~min})\end{array}$ & $\begin{array}{l}\text { Sample inlet } \\
\text { flow/frit flow } \\
Q_{\mathrm{s}} / Q_{\mathrm{f}}\end{array}$ \\
\hline 1 & 1.5 & 0.13 & 16.0 & 0.13 \\
2 & 3.0 & 0.13 & 29.0 & 0.07 \\
3 & 4.0 & 0.13 & 37.4 & 0.05 \\
4 & 4.5 & 0.13 & 41.7 & 0.04
\end{tabular}

${ }^{a} t_{1}+t_{\mathrm{p}}$ i.e. the time for relaxation and linear decay of crossflow. 

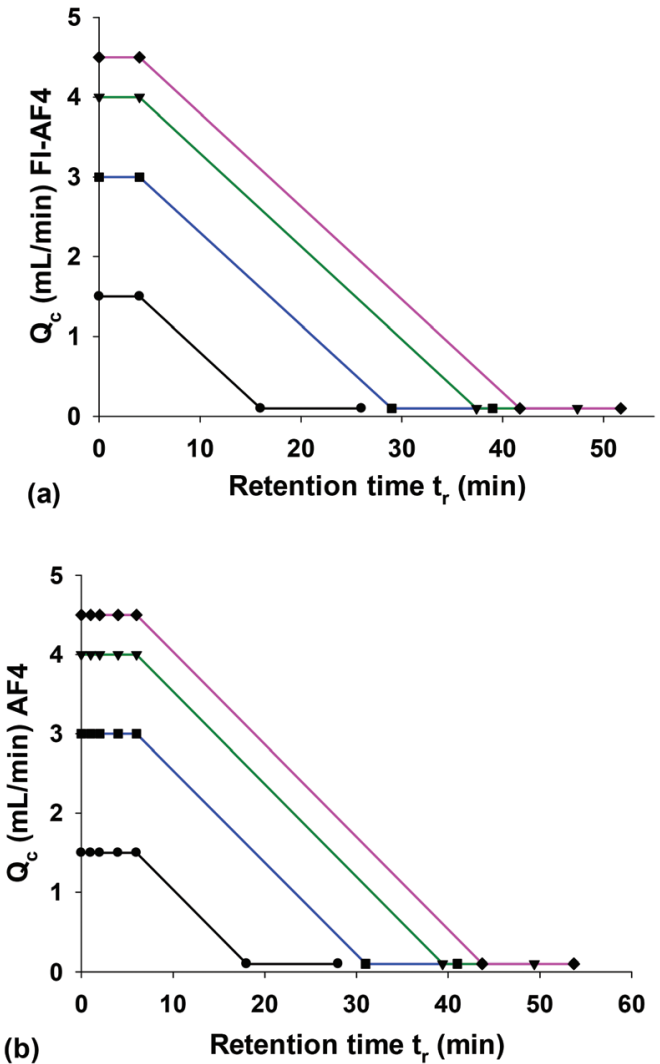

Fig. 1 Crossflow programming profiles used for studying the effect of initial crossflow rate $\left(Q_{\mathrm{c0}}\right)$ in FI-AF4 (a) and AF4 (b) following eqn (1) and (2), respectively.

to the focusing/relaxation step. In the case of FI-AF4, this time corresponds to the hydrodynamic relaxation time. After that, the elution started according to the programing conditions described in Table 1, where the $Q_{\text {cf }}$ was kept constant for $10 \mathrm{~min}$. The channel was then flushed without any crossflow for $10 \mathrm{~min}$ before the next injection.

\section{The effect of channel flow ( $\left.Q_{\text {out }}\right)$}

In order to study the effect of $Q_{\text {out }}$ on the mass recovery and resolution $\left(R_{\mathrm{S}}\right)$ in the conventional $\mathrm{AF} 4$ and FI-AF4 channels, $Q_{\text {out }}$ was varied from 0.2 to $1.0 \mathrm{~mL} \mathrm{~min}^{-1}$. All other run conditions were the same as those described in the previous section.

\section{The effect of the initial crossflow $\left(Q_{\mathrm{co}}\right)$ at constant $Q_{\mathrm{co}} / Q_{\text {out }}$}

To examine the effect of $Q_{\mathrm{co}}$ on the mass recovery and resolution $\left(R_{\mathrm{S}}\right)$, at constant $Q_{\mathrm{c} 0} / Q_{\text {out }}$ of 4.5 , three different crossflow rates were applied: $1.5,3.0$ and $4.5 \mathrm{~mL} \mathrm{~min}^{-1}$. In order to keep the ratio of $Q_{\mathrm{co}} / Q_{\text {out }}$ constant, the $Q_{\text {out }}$ was set at $0.33,0.66$ and $1.00 \mathrm{~mL} \mathrm{~min}^{-1}$, respectively. All other run conditions were the same as those described above.

\section{Data analysis and processing}

Data obtained from MALS and dRI detectors were recorded and processed using the Astra software (v. 6.1.7, Wyatt
Technology). $M$ and $r_{\text {rms }}$ values were obtained from a $1^{\text {st }}$ order fitting of the MALS data obtained from the detectors 6-15 (scattering angles of $42.8^{\circ}-132.2^{\circ}$ ) using the Berry method. ${ }^{14,15}$ The second virial coefficient $A_{2}$ for all samples was assumed to be negligible. All analyses were performed in triplicate, to ensure reproducibility.

The comparison between AF4 and FI-AF4 was summarized using a projection to latent structures (PLS) regression by means of partial least square analysis (Matlab R2017b, v.9.3.0.713579, The MathWorks, Inc., USA). The input variables were: initial crossflow $\left(Q_{\mathrm{c} 0}\right)$, relaxation time and channel flow $\left(Q_{\text {out }}\right)$. The output variables were: resolution $\left(R_{\mathrm{s}}\right)$ and mass recovery (\%).

\section{Theory/calculations}

\section{Mass recovery}

The absolute sample recovery is defined as the percentage of an injected component or sample that is recovered from the separation system. ${ }^{1}$ It may not be easy to measure because it is necessary to quantitatively determine the amount of each sample component (not only the entire sample) that is introduced into the system. One way is bypassing the channel and measuring the concentration of the components. Another way is to compare the dRI signal area between runs obtained with and without applying crossflow $\left(Q_{\mathrm{c}}\right)$. This can be expressed by the following equation:

$$
\text { Sample recovery }(\%)=\frac{\text { Mass determined }}{\text { Mass injected }} \times 100 \%
$$

where mass determined is the mass eluted from the separation channel, calculated from the integration of the dRI signal (after subtraction of the baseline from a blank run obtained at the same conditions as those used for the sample). The subtraction of the baseline is necessary to avoid any changes produced by differences in pressure during the runs when crossflow decaying with time is applied. The mass injected is the mass eluted from the separation channel calculated from the integration of the dRI signal with no crossflow applied. In the present study, the mass recovery was determined using eqn (1), and all analyses were performed in triplicate to ensure reproducibility.

\section{Plate height}

The plate height $(H)$ is the height (or length) of a theoretical stage or zone established after a substance or mixture of substances reaches an equilibrium in a separation system. Usually, the performance of a separation system improves as $H$ decreases due to reduced zone broadening. $H$ is defined as the variance $\sigma^{2}$ of the concentration profile divided by the mean displacement $(Z)$ of the profile.

$$
H=\frac{\sigma^{2}}{Z}
$$


$Z$ can be identified by the channel length $L,{ }^{16}$ and $H$ can be determined experimentally by

$$
H=\frac{L \sigma_{t}^{2}}{t_{\mathrm{r}}^{2}}
$$

where $\sigma_{t}$ is the peak standard deviation in time units and $t_{\mathrm{r}}$ is the retention time $\left(t_{\mathrm{r}}\right)$.

\section{Resolution}

Resolution $\left(R_{\mathrm{S}}\right)$ is a measure of separation between two peaks. $R_{\mathrm{S}}$ can be calculated by

$$
R_{\mathrm{s}}=\frac{\Delta t_{\mathrm{r}}}{\bar{w}_{\mathrm{b}}}
$$

where $\Delta t_{\mathrm{r}}$ is the difference in retention times between two peaks and $\bar{w}_{\mathrm{b}}$ is the average of the peak widths. Assuming a peak is Gaussian with the standard deviation, $\sigma$, the peak width at the half-height $\left(w_{1 / 2 h}\right)$ is $2.354 \sigma$, and $R_{\mathrm{S}}$ can be determined by

$$
R_{\mathrm{s}}=1.18 \times\left(\frac{\Delta t_{\mathrm{r}}}{w_{1 / 2 h 1}+w_{1 / 2 h 2}}\right)
$$

\section{Results and discussion}

\section{Effect of sample concentration in AF4}

In a previous study, ${ }^{11}$ the same polymers as in the present study were analysed: either through individual injected polymer samples or as an injected mixture of the same two samples. A shift in $t_{\mathrm{r}}$ of peak maxima of both the Rayleigh ratio and the dRI signal was observed after mixing. In order to avoid this possible shift in $t_{\mathrm{r}}$, especially for the analysis of $H$ and $R_{\mathrm{s}}$, samples prepared with different weight ratios (Table 1) were injected to find a constant $t_{\mathrm{r}}$ in the mixture. Fig. 2 shows the AF4 fractograms (from MALS and dRI detector) of the mixtures obtained either using the conventional AF4 channel (Fig. 2(a and b)) or an FI-AF4 channel (Fig. 2(c and d)). In both AF4 and FI-AF4 channels, the intensity of the dRI signals were proportional to the sample concentration in the mixture. In addition, the MALS signal at $90^{\circ}$ scattering angle for AF4 channel is shown in Fig. 2(a), where the peak maximum of glycogen injected alone was $t_{\mathrm{r}}=15.6 \mathrm{~min}$ and for pullulan injected alone, the peak maximum was $t_{\mathrm{r}}=36.0 \mathrm{~min}$. As shown in Fig. 2(a), the peak maximum $t_{\mathrm{r}}$ for glycogen gradually increases as the relative content of pullulan increases in the mixture, while the peak maximum $t_{\mathrm{r}}$ for pullulan gradually decreases with increasing relative content of glycogen in the mixture. When the mass ratio is in the range of $1: 4$ to $1: 6$, the $t_{\mathrm{r}}$ of peak maxima of glycogen and pullulan in the mixture remained constant at $17.7 \mathrm{~min}$ and $34.0 \mathrm{~min}$, respectively. Similar results were observed for the FI-AF4 channel (Fig. 2(c and $\mathrm{d})$ ), i.e. peak maximum $t_{\mathrm{r}}$ for glycogen injected alone at $11.8 \mathrm{~min}$ and peak maximum $t_{\mathrm{r}}$ for pullulan injected alone was $34.8 \mathrm{~min}$ (MALS signals). The peak maximum $t_{\mathrm{r}}$ for glycogen gradually increases as the relative content of pullulan increases in the mixture, while the peak maximum $t_{\mathrm{r}}$ of pullulan gradually decreases with increasing relative content of the glycogen in the mixture. Similarly, to the AF4-results, at mass ratio $1: 4$ to $1: 6$, the peak maxima of $t_{\mathrm{r}}$ for glycogen and pullulan, in the mixture, remain constant.

The shift in $t_{\mathrm{r}}$ of the peak maxima from the MALS and dRI signals in the mixtures, compared with the individual polymers (i.e. glycogen and pullulan) was also observed in our previous work. ${ }^{11}$ It was suggested, that this effect could likely be due to interactions between the polymer molecules, which may influence the relaxation, and establishment of a concentration profile. Consequently, retention times would also be affected. Polymer interactions would be particularly important during the focusing/relaxation step in the analysis as this generates a sample zone of higher concentration than the original sample. In the current study, the same behaviour was observed when the focusing/relaxation step was omitted using the FI-AF4 channel. To ensure that these changes in peak maxima are not due to incomplete resolution in the separation, a deconvolution analysis of the fractograms of the mixtures was performed (data not shown). However, the same tendency of shifts was observed in the deconvoluted fractograms.

Incompatibility of different polymers in solution is well known, especially at somewhat higher concentrations. The incompatibility eventually leads to segregative phase separation, in which the different phases are enriched in one of the polymers. For mixtures of non-ionic polymers in aqueous solution, as in the current study, segregation is the rule and the tendency towards segregation becomes stronger with increasing molar mass of either of the polymer components. ${ }^{17}$ Relatively small repulsive interaction between the different polymers, or differences in the polymer-solvent interaction, will result in segregation. A classic example of segregation is the mixture of dextran and poly(ethylene oxide), which has been widely used for the partition of biological polymers. ${ }^{18}$

In the current study, the chemical differences between the polymers are subtler, i.e. both polymers are glucans but with differently linked glucose units. In addition, pullulan is linear while glycogen is hyper-branched (average degree of branching $=7.4-9.2) .{ }^{19}$ Segregation is reported to happen in non-ionic polymer mixtures of pullulan and dextran, which have some similarity to the current case as the mixture contains a linear and a branched glucan. ${ }^{20}$ Segregation was observed at higher polymer molar mass: $M_{\mathrm{w}} \geq 2.6 \times 10^{5} \mathrm{~g} \mathrm{~mol}^{-1}$ for pullulan and $M_{\mathrm{w}} \geq 6.2 \times 10^{5} \mathrm{~g} \mathrm{~mol}^{-1}$ for dextran. These weight-average molar mass values are lower than for the pullulan $\left(M_{\mathrm{W}}=2.1 \times\right.$ $\left.10^{5} \mathrm{~g} \mathrm{~mol}^{-1}\right)$ and glycogen $\left(M_{\mathrm{W}}=2.9 \times 10^{5} \mathrm{~g} \mathrm{~mol}^{-1}\right)$ used in the current study. Thus, the shift in $t_{\mathrm{r}}$ could be related to an incompatibility of the polymers in the mixture, which influences the elution when the concertation is increased in the sample zone.

\section{Effect of initial crossflow $\left(Q_{\mathrm{co}}\right)$ and focus/relaxation time on mass recovery, plate height and resolution}

The effect of $Q_{\mathrm{co}}$ and focus/relaxation time were studied in a conventional AF4 channel and an FI-AF4 channel at the 

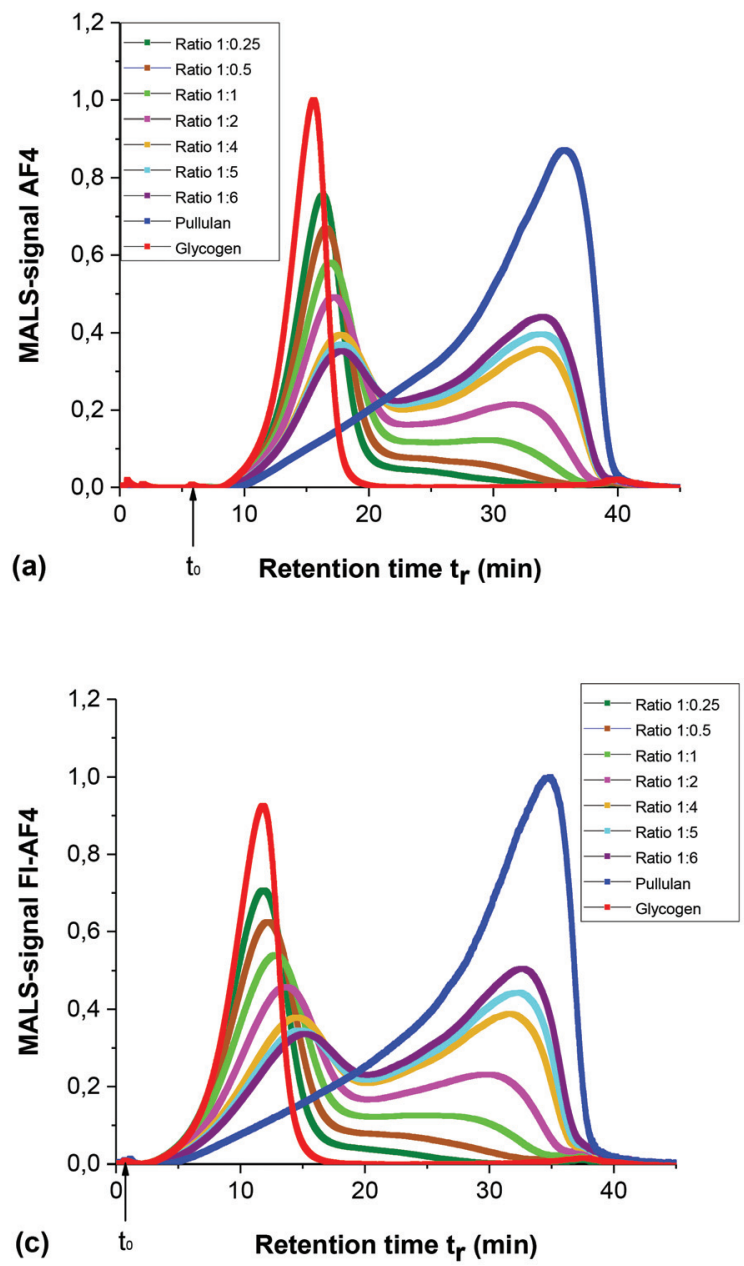
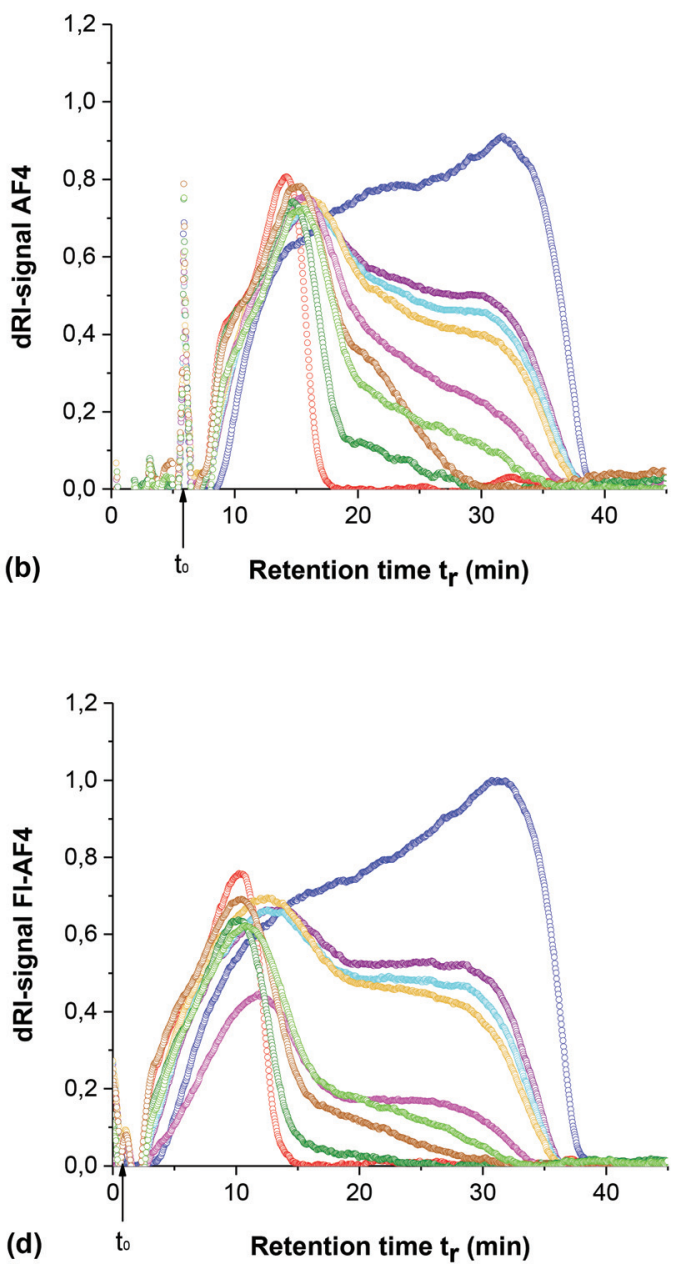

Fig. 2 Fractograms of individual solutions and mixtures of glycogen and pullulan obtained from AF4 (a-b) and FI-AF4 (c-d) channel. Fractograms (a) and (c) show the MALS-signal at $90^{\circ}$ scattering angle and fractograms (b) and (d) show the dRI-signal.

channel flow rate $Q_{\text {out }}$ of 1 and $0.2 \mathrm{~mL} \mathrm{~min}^{-1}$, respectively. In the case of the FI-AF4 channel, the $Q_{\text {out }}$ was set to $0.2 \mathrm{~mL} \min ^{-1}\left(Q_{\mathrm{s}}=Q_{\text {out }}\right)$ to ensure that the entire frit flow $\left(Q_{\mathrm{f}}\right)$ could be used as the crossflow $\left(Q_{\mathrm{c}}\right)$. It has been demonstrated in the analysis of proteins that higher resolution is obtained when $Q_{\mathrm{s}}=Q_{\text {out }}$, although the analysis time is prolonged. ${ }^{4}$

Fig. 3 shows the mass recovery $(\mathrm{a}-\mathrm{b})$, plate height $(\mathrm{c}-\mathrm{d})$, and resolution ( $\mathrm{e}-\mathrm{f}$ ) determined for a mixture of glycogen and pullulan in the AF4 and FI-AF4 channels as a function of the initial crossflow rate $\left(Q_{\mathrm{co}}\right)$. In the AF4 channel (Fig. 3a), at $Q_{\mathrm{c} 0}$ of $0.5 \mathrm{~mL} \mathrm{~min}^{-1}$, the mass recoveries were $90 \%$ and $95 \%$, at focus times of 2 and $4 \mathrm{~min}$, respectively. As $Q_{\mathrm{co}}$ increases, the mass recovery decreases somewhat, to about $85 \%$. In the FI-AF4 channel (Fig. 3b), all sample recoveries are higher and no significant changes were observed with $Q_{\mathrm{c} 0}$ or the relaxation time. It should be noted that the mass recoveries in the FI-AF4 channel (about 105\%) are higher than those in the conventional AF4 channel.

In Fig. 3c-f, no data is shown for the focus/relaxation time of $2 \mathrm{~min}$ as no separation between the glycogen and pullulan was observed in either channel (elution occurred as one broad peak). In the AF4 channel (Fig. 3c), as $Q_{\mathrm{co}}$ increases, the plate height $(H)$ of the first peak (corresponding to glycogen red line Fig. 2a) increases (from 0.20 to 0.75 ), while that of the second peak (corresponding to pullulan blue line Fig. 2a) decreases (from 0.30 to 0.05 ). In the FI-AF4 channel (Fig. 3d), no separation between glycogen and pullulan was observed at $Q_{\mathrm{c} 0}=$ $1.5 \mathrm{~mL} \mathrm{~min}^{-1}$. In addition, as $Q_{\mathrm{co}}$ increases, the plate height of both peaks decreased (from 0.9 to 0.75 and from 0.1 to 0.04 for the glycogen and pullulan, respectively).

In Fig. $3 \mathrm{e}$ and $\mathrm{f}$, the resolution increases with increasing $Q_{\text {co }}$ in both channels (from 0.7 to 1.9 and from 0.4 to 0.9 in the conventional AF4 and FI-AF4 channels, respectively). At the same $Q_{\mathrm{c} 0}$, the resolution in the AF4 channel was higher (almost twice) than the resolution in the FI-AF4 channel. No significant differences in $R_{\mathrm{S}}$ were observed for 4-6 min relaxation time in both channels.

\section{Effect of channel flow ( $\left.Q_{\text {out }}\right)$ on mass recovery and resolution}

To study the effect of $Q_{\text {out }}$ on the mass recovery and $R_{\mathrm{s}}, Q_{\text {out }}$ was varied $\left(0.2-1.0 \mathrm{~mL} \mathrm{~min}^{-1}\right)$ with $Q_{\mathrm{c} 0}$ kept constant at $1.5 \mathrm{~mL} \min ^{-1}$ for a mixture of glycogen and pullulan. Fig. $4 \mathrm{a}$ 

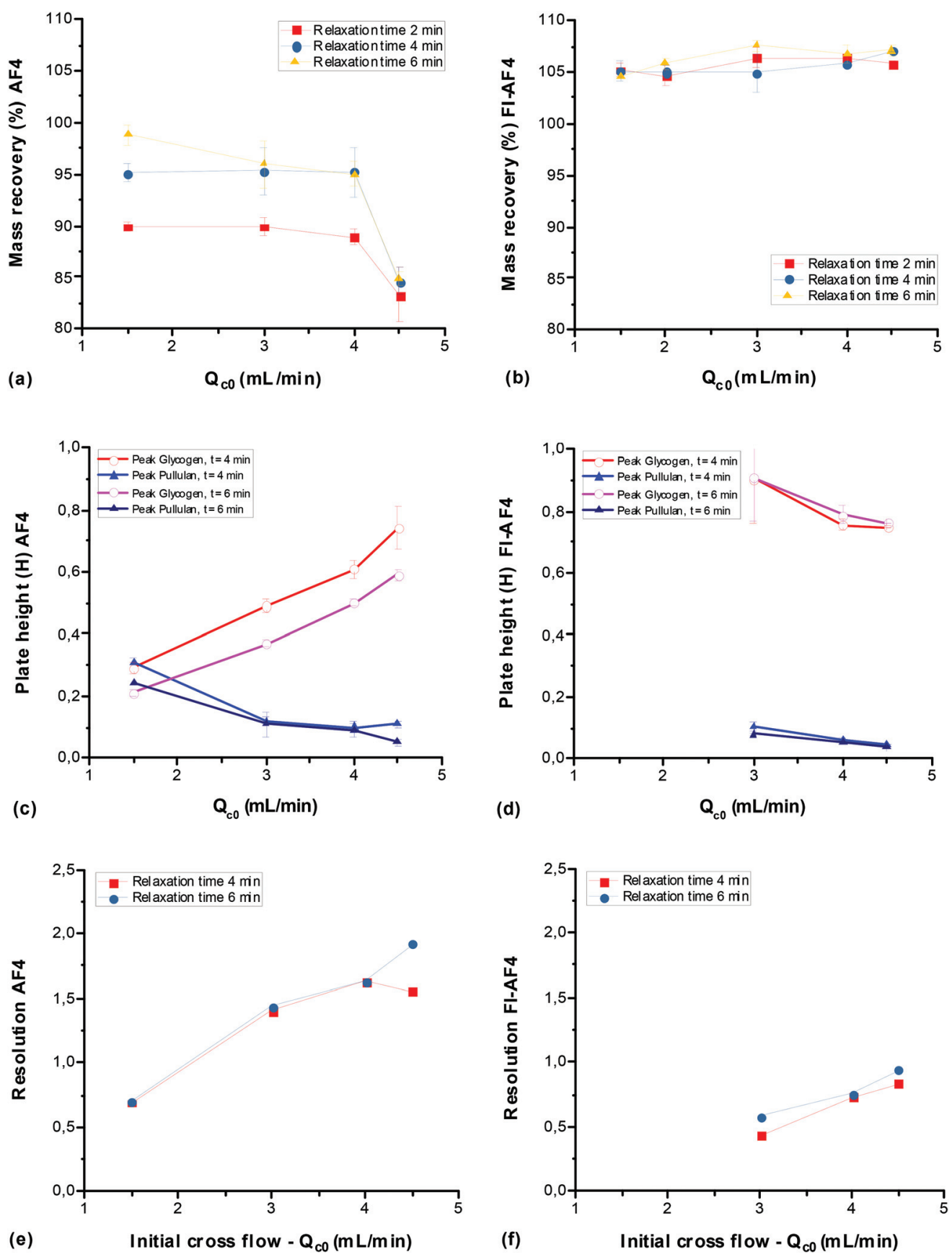

Fig. 3 The effect of initial crossflow rate $\left(Q_{\mathrm{co}}\right)$ and relaxation time on: mass recovery (\%) obtained at constant channel flow $\left(Q_{\text {out }}\right)$ of $1 \mathrm{~mL}$ min ${ }^{-1}$ in conventional AF4 channel (a), and $0.2 \mathrm{~mL} \mathrm{~min}^{-1}$ in FI-AF4 channel (b); plate height $(H)$ of conventional AF4 channel (c), and FI-AF4 channel (d) and resolution $\left(R_{\mathrm{s}}\right)$ of AF4 channel (e) and FI-AF4 channel ( $\mathrm{f}$ ). The error bars are the standard deviation of three measurements.

shows the mass recovery and Fig. 4b the resolution measured at various $Q_{\text {out }}$. Results from the AF4 and FI-AF4 channels are presented in red and blue, respectively. As shown in Fig. 4a, the mass recoveries were higher than $95 \%$ in all cases, which suggests that there was no significant sample loss during the analyses. As $Q_{\text {out }}$ increased, the recovery gradually decreased somewhat in both channels. No significant differences were observed between the mass recoveries in the AF4 and the FI-AF4 channels, although the recoveries in the AF4 channel tended to be slightly higher than those in the FI-AF4 channel. Fig. $4 \mathrm{~b}$ shows that $R_{\mathrm{S}}$ increases with increasing $Q_{\text {out }}$ in both channels. At $Q_{\text {out }} \geq 0.8 \mathrm{~mL} \mathrm{~min}^{-1}$, the increase was higher in the AF4 channel (from 1.40 to 1.65 ) than in the FI-AF4 (from 1.15 to 1.2).

\section{Effect of initial crossflow $\left(Q_{\mathrm{co}}\right)$ on mass recovery and resolution} at constant $Q_{\mathrm{co}} / Q_{\text {out }}$

To study the effect of $Q_{\mathrm{c} 0}$ on the mass recovery and $R_{\mathrm{s}}, Q_{\mathrm{co}}$ was varied (1.5-4.5 $\mathrm{mL} \mathrm{min}^{-1}$ ) for the mixture of glycogen and pull- 

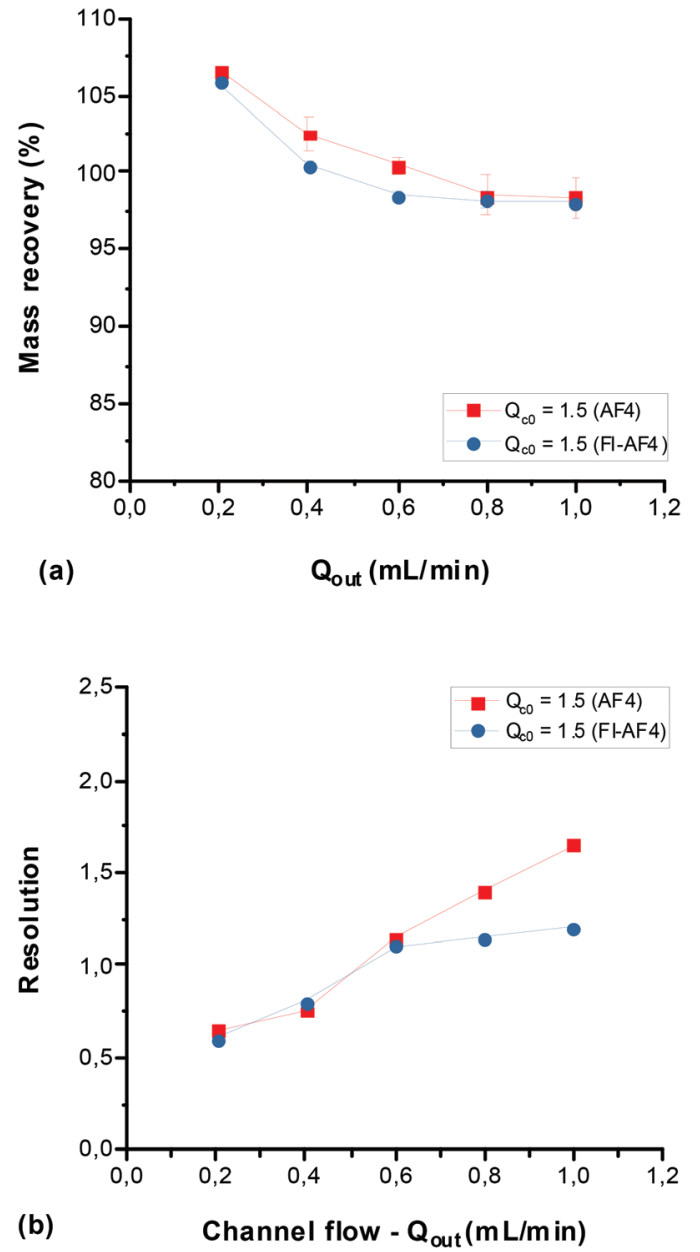

Fig. 4 Effect of channel flow $\left(Q_{\text {out }}\right)$ at constant initial crossflow $\left(Q_{\mathrm{cO}}\right)$ of

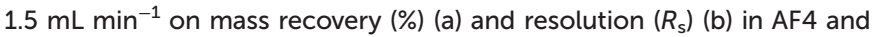
FI-AF4 channels. The error bars are the standard deviation of three measurements.

ulan. For a comparison of the two channels (AF4 and FI-AF4), the ratio $Q_{\mathrm{c} 0} / Q_{\text {out }}$ was kept constant at $4.6 \mathrm{~mL} \mathrm{~min}^{-1}$, giving the same retention level. This allows for a more straightforward comparison, especially in terms of $R_{\mathrm{S}}$. Moreover, the results from the conventional and FI-AF4 channels are presented in red and blue, respectively. Fig. 5a shows that, as $Q_{\mathrm{co}}$ increased, the mass recovery in the AF4 channel decreased from $100 \%$ to $84 \%$ while in the FI-AF4 channel, it increased from $96 \%$ to $97.5 \%$. Thus, at the highest $Q_{\mathrm{co}}$, the mass recovery in the FI-AF4 channel is higher than in the AF4 channel. Fig. 5b shows that $R_{\mathrm{S}}$ increased as $Q_{\mathrm{co}}$ increased in both channels. The increase in $R_{\mathrm{S}}$ was significantly higher in the conventional AF4 channel (from 0.56 to 2.10) than in the FI-AF4 channel (from 0.27 to 0.7 ).

To summarize the results for the comparison between AF4 and FI-AF4, a PLS analysis was performed. The PLS analysis is used to find a linear regression model by projecting the input variables and the output variables to a new space. Hence, from this model allows to make general predictions on how output variables are influenced by input variables. The PLS plot is
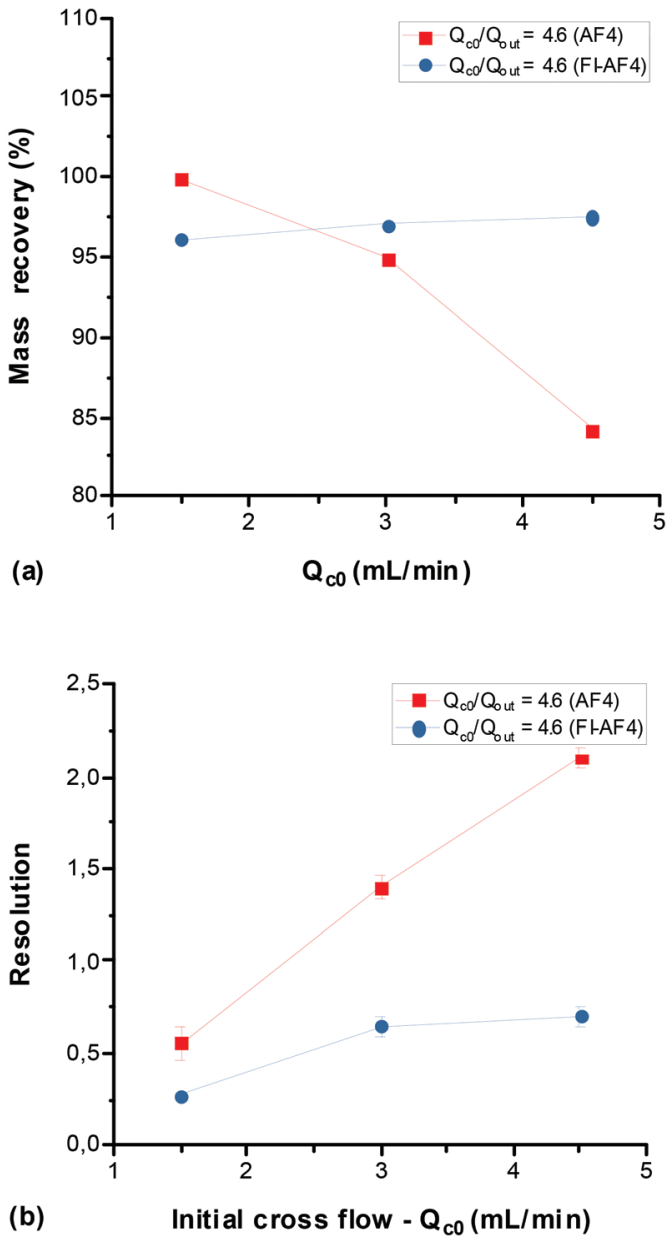

Fig. 5 Effect of initial crossflow rate $\left(Q_{\mathrm{co}}\right)$ at constant $Q_{\mathrm{c0}} / Q_{\text {out }}$ of 1.5 on mass recovery (\%) (a) and resolution $R_{\mathrm{s}}$ (b) in AF4 and FI-AF4 channels. The error bars are the standard deviation of three measurements.

shown in Fig. 6. It is possible to conclude that the percentage of mass recovery (\%) is higher when using the FI-AF4 channel in comparison with the conventional AF4 channel. Furthermore, there is an opposite relationship between mass recovery (\%) and $Q_{\text {out }}$. The $R_{\mathrm{S}}$ is higher in the $\mathrm{AF} 4$ channel in comparison with the FI-AF4 channel, and it seems that there is a correlation $\left(r^{2}=\right.$ $0.63)$ between the relaxation time and resolution $\left(R_{\mathrm{s}}\right)$.

\section{Overloading effect using waxy maize starch}

WM starch was utilized for investigating the effect of injected mass on overloading effects of ultra-high molar mass branched polymers. In Fig. 7a and b, MALS signal at $90^{\circ}$ scattering angle fractograms are shown from AF4 and FI-AF4 channels. Different concentrations of samples were injected from $0.125 \mathrm{mg} \mathrm{mL}^{-1}$ to $1.000 \mathrm{mg} \mathrm{mL}^{-1}$, which in terms of injected mass were $1.25 \mu \mathrm{g}$ to $10.0 \mu \mathrm{g}$. The results from the AF4 channel show that the peak maxima $t_{\mathrm{r}}$ was constant at approx. $32 \mathrm{~min}$ at the two lowest concentrations (i.e. injected mass 1.25 and $2.5 \mu \mathrm{g}$ ). At higher concentrations, $t_{\mathrm{r}}$ gradually decreases with increasing sample concentration (Fig. 7a), demonstrating that overloading occurs. The overloading can 


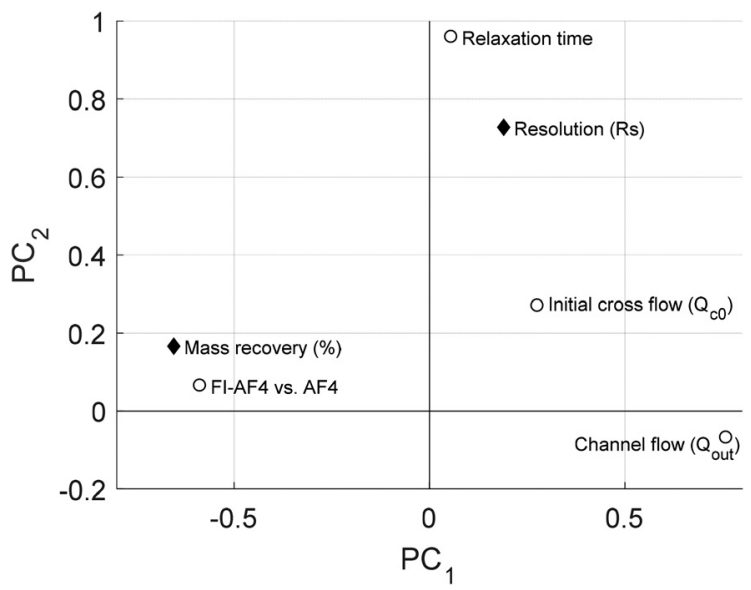

Fig. 6 PLS analysis comparing FI-AF4 vs. AF4 channels. Income variables: initial crossflow rate $\left(Q_{c 0}\right)$, channel flow $\left(Q_{\text {out }}\right)$ and relaxation time. Output variables: mass recovery $(\%)$ and resolution $\left(R_{\mathrm{s}}\right)$. The FI-AF4 vs. AF4 is a dummy input variable.
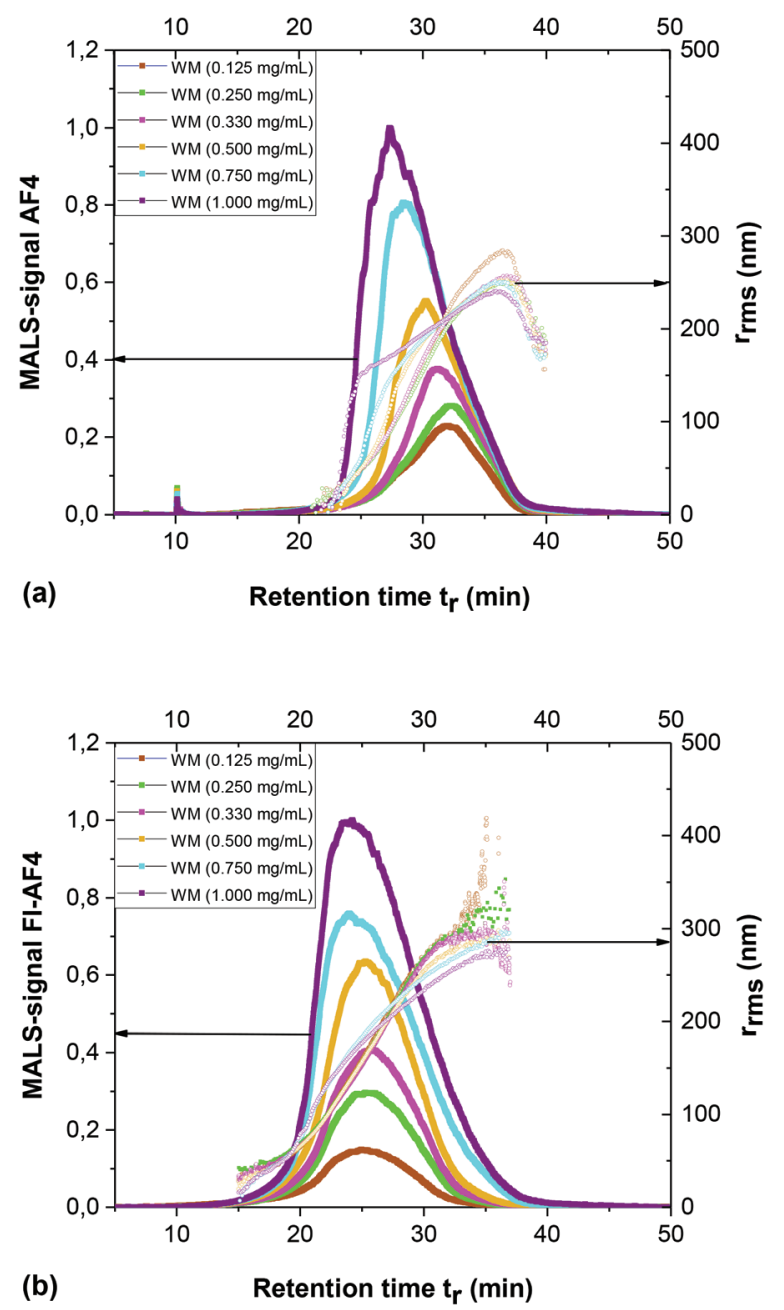

Fig. 7 Effect of overloading on WM. Fractograms show the MALS signal at $90^{\circ}$ scattering angle of WM from conventional AF4 channel (a) and FI-AF4 channel (b). be also noted by the change in the slope trend of $r_{\text {rms }} v s . t_{\mathrm{r}}$ (Fig. 7a). Similar results for WM starch were reported previously, ${ }^{7,8}$ where overloading occurs at a similar injected amount of sample $(\geq 2 \mu \mathrm{g})$.

For the FI-AF4 channel, the results are shown in Fig. $7 \mathrm{~b}$. The $t_{\mathrm{r}}$ of peak maxima remains constant until a concentration of $0.50 \mathrm{mg} \mathrm{mL}^{-1}$ (i.e. injected mass of $5.0 \mu \mathrm{g}$ ) and the slope in $r_{\text {rms }} v s . t_{\mathrm{r}}$ also remains unchanged, suggesting there exists no significant overloading. Additionally, it can be observed that even when the higher injected amounts cause overloading (i.e. shift in peak maxima) the selectivity on $r_{\text {rms }}$ remains higher compared to AF4. Thus, even though overloading occurs its consequences seems less severe in FI-AF4.

In addition, overloading is observed starting at $0.75 \mathrm{mg} \mathrm{mL}^{-1}$ (i.e. injected mass of $7.5 \mu \mathrm{g}$ ). In short, the sample injection mass was twice as high in the FI-AF4 channel as the mass injected in the AF4 channel, without observed overloading.

\section{Conclusion}

In this study, performances of a conventional AF4 and an FI-AF4 channel were compared in terms of mass recovery, plate height $(H)$ and resolution $\left(R_{\mathrm{s}}\right)$ using a mixture of biopolymers (glycogen and pullulan) that have overlapping molar mass $(M)$ range but different hydrodynamic size and structure (branching or linear). Additionally, differences in the overloading effect were examined using waxy maize starch (WM). The results in this study show that the relaxation time in both the $\mathrm{AF} 4$ and the FI-AF4 channels (i.e. focusing or hydrodynamic relaxation respectively) does not have a considerable effect on the separation channel mass recovery. The relaxation time does correlate with resolution $\left(R_{\mathrm{s}}\right)$. The mass recovery (\%) in the FI-AF4 channel is higher in comparison with the AF4 channel. When the same $Q_{\text {out }}$ is applied, the mass recoveries in the two channels were similar. The $R_{\mathrm{S}}$ was higher for AF4, suggesting that without regard to running conditions, the conventional AF4 channel yields higher $R_{\mathrm{S}}$ than the FI-AF4 channel for the analysis of the biopolymers. In addition, $R_{\mathrm{S}}$ increased as either $Q_{\mathrm{co}}$ or $Q_{\text {out }}$ increased in both channels as is expected. Furthermore, it was possible to inject higher concentrations of waxy maize starch (i.e. twice the injected mass) onto the FI-AF4 compared to the conventional AF4 channel without observing overloading effects.

\section{Conflicts of interest}

The authors declare that they have no potential conflict of interest.

\section{Acknowledgements}

Swedish International Development Agency (SIDA) in a collaborative project between the Higher University of San Andres UMSA (Bolivia) and Lund University (Sweden) supported 
the present study. National Research Foundation (NRF) of Korea (NRF-2013K2A3A1000086 and NRF-2019R1A2C1006677) in cooperation with the Swedish Foundation for International Cooperation in Research and Higher Education (STINT). Dr Andreas Håkansson, Dept. of Food Technology, Engineering and Nutrition, Lund University is gratefully acknowledged for assistance with the PLS analysis.

\section{Notes and references}

1 J. C. Giddings, in Field-Flow Fractionation Handbook, ed. M. E. Schimpf, K. Caldwell and J. C. Giddings, Wiley New York, 2000, pp. 4-27.

2 K. G. Wahlund and J. C. Giddings, Anal. Chem., 1987, 59, 1332-1339.

3 K.-G. Wahlund and L. Nilsson, in Field-Flow Fractionation in Biopolymer Analysis, ed. S. K. R. Williams and K. D. Caldwell, Springer Vienna, Vienna, 2012, pp. 1-21, DOI: 10.1007/978-3-7091-0154-4_1.

4 M. H. Moon, H. Kwon and I. Park, J. Liq. Chromatogr. Relat. Technol., 1997, 20, 2803.

5 M. K. Liu, P. S. Williams, M. N. Myers and J. C. Giddings, Anal. Chem., 1991, 63, 2115-2122.

6 M. H. Moon, H. Kwon and I. Park, Anal. Chem., 1997, 69, 1436-1440.
7 M. Van Bruijnsvoort, K. G. Wahlund, G. Nilsson and W. T. Kok, J. Chromatogr. A, 2001, 925, 171-182.

8 D. Perez-Rea, B. Bergenståhl and L. Nilsson, Anal. Bioanal. Chem., 2015, 407, 4315-4326.

9 M. H. Moon and I. Hwang, J. Liq. Chromatogr. Relat. Technol., 2001, 24, 3069.

10 M. H. Moon and P. S. Williams, Anal. Chem., 1999, 71, 2657.

11 C. Zielke, C. Fuentes, L. Piculell and L. Nilsson, J. Chromatogr. A, 2018, 1532, 251-256.

12 C. Fuentes, D. Perez-Rea, B. Bergenståhl, S. Carballo, M. Sjöö and L. Nilsson, Int. J. Biol. Macromol., 2019, 125, 829-838.

13 L. Nilsson, Food Hydrocolloids, 2013, 30, 1-11.

14 G. C. Berry and J. Chem, Phys., 1966, 44, 4550-4564.

15 M. Andersson, B. Wittgren and K.-G. Wahlund, Anal. Chem., 2003, 75, 4279-4291.

16 J. C. Giddings, Unified Separation Science, Wiley, New York, 1991.

17 L. Piculell and B. Lindman, Adv. Colloid Interface Sci., 1992, 41, 149-178.

18 P.-Å. Albertsson, Partition of cell particles and macromolecules, Wiley, New York, 3rd edn, 1986, 346 pp.

19 C. Fernandez, C. C. Rojas and L. Nilsson, Int. J. Biol. Macromol., 2011, 49, 458-465.

20 A. Eckelt, J. Eckelt, W. Schärtl and B. A. Wolf, Macromol. Chem. Phys., 2012, 213, 1206-1215. 